\title{
INDEX TO THE BIBLIOGHRAPHY, SUBJECTS AND COUNTRIES
}

GENERAL ISSUES

America, 277

Brazil, 277

Social Theory and Social Science, Chile, 277f.

88f., 180ff., $271 \mathrm{ff}$.

History, 89ff., 181ff., $273 \mathrm{ff}$.

Contemporary Issues, 186, 276f,

\section{CONTINENTS AND COUNTRIES}

Africa, 92, $186 \mathrm{f}$.

Egypt, 187
Mexico, 278

Surinam, 93
Asia
China, 189, $281 \mathrm{f}$.
India, $94 \mathrm{f}$.
Israel, 95
Laos, 282

Europe, 189, $282 \mathrm{ff}$.

Austria, 95f., 190, 284

Belgium, 284f.

Bulgaria, 96

Czechoslovakia, 96, 285
France, $96 \mathrm{ff}$, , 190ff., $285 \mathrm{ff}$.

Germany, 98ff., 192ff., $287 \mathrm{ff}$.

Great Britain, 101ff., 196f., $297 \mathrm{ff}$.

Greece, 104

Italy, 198f., 300

The Netherlands, 104,300

Spain, 300f.

Union of Soviet Socialist Republics - Russia, 105, 199f., 301 